\title{
On Solving Centrosymmetric Linear Systems
}

\author{
Moawwad El-Mikkawy", Faiz Atlan \\ Mathematics Department, Faculty of Science, Mansoura University, Mansoura, Egypt \\ Email: ${ }^{*}$ _elmikkawy@yahoo.com, faizatlan11@yahoo.com
}

Received November 2, 2013; revised December 2, 2013; accepted December 9, 2013

Copyright (C) 2013 Moawwad El-Mikkawy, Faiz Atlan. This is an open access article distributed under the Creative Commons Attribution License, which permits unrestricted use, distribution, and reproduction in any medium, provided the original work is properly cited. In accordance of the Creative Commons Attribution License all Copyrights (C) 2013 are reserved for SCIRP and the owner of the intellectual property Moawwad El-Mikkawy, Faiz Atlan. All Copyright (C) 2013 are guarded by law and by SCIRP as a guardian.

\begin{abstract}
The current paper is mainly devoted for solving centrosymmetric linear systems of equations. Formulae for the determinants of tridiagonal centrosymmetric matrices are obtained explicitly. Two efficient computational algorithms are established for solving general centrosymmetric linear systems. Based on these algorithms, a MAPLE procedure is written. Some illustrative examples are given.
\end{abstract}

Keywords: Centrosymmetric Matrix; Exchange Matrix; Rotate Matrix; Linear System; DETGTRI Algorithm; Computer Algebra Systems (CAS); MAPLE

\section{Introduction}

Throughout this paper, $|A|$ and $A^{\mathrm{T}}$ denote the determinant and the transpose of the matrix $A$ respectively. Also $\lfloor x\rfloor$ denotes the greatest integer less than or equal to $x$. Centrosymmetric matrices have practical applications in numerical analysis, information theory, statistics, physics, harmonic differential quadrature, differential equations, engineering, sinc methods, magic squares, linear system theory and pattern recognition. The interested readers may refer to [1-12].

Solving and analyzing linear systems of equations is a fundamental problem in science and engineering applications. The cost of solving any linear system using Gauss or Gauss-Gordan algorithms is $O\left(n^{3}\right)$. The motivation of the current paper is to develop efficient algorithms for solving any centrosymmetric linear system having $n$ equations and $n$ unknowns provided that the coefficient matrix of the system is nonsingular. The cost of each algorithm depends on the solvers of two associated linear systems having smaller sizes than $n$. More precisely, if $n$ $=2 \mathrm{~m}$, then each of the two associated linear systems consists of $m$ equations. If $n=2 m+1$, then we have one system having $\mathrm{m}$ equations and the other has $(m+1)$ equations. Consequently, if the two associated linear systems have special structures, then the cost of the centrosymmetric algorithm could be considerably reduced, in particular for large values of $n$.

*Corresponding author.
The paper is organized as follows. In Section 2, some properties of the exchange and the rotate matrices are presented. Formulae for centrosymmetric tridiagonal determinants are obtained in Section 3. In Section 4, two computational algorithms for solving centrosymmetric linear systems are given. A MAPLE procedure is given in Section 5. Some illustrative examples are presented in Section 6.

Definition 1.1. The $n \times n$ matrix with 1 's on the northeast-southeast diagonal and 0 's elsewhere

$$
J_{n}=\left[\begin{array}{ccccc}
0 & \cdots & \cdots & 0 & 1 \\
\vdots & & . & 1 & 0 \\
\vdots & . & . & . & \vdots \\
0 & 1 & . & & \vdots \\
1 & 0 & \cdots & \cdots & 0
\end{array}\right],
$$

is called the exchange matrix of order $n$. The subscript on $J_{n}$ is neglected whenever the size is obvious from the context.

Definition 1.2. Let $A=\left(a_{i j}\right)_{i, j=1}^{n}$ be an $n \times n$ matrix. The rotate of $A$, denoted $A^{R}$, is defined by

$$
A^{R}=J A J .
$$

Definition 1.3 [9]. Let $A=\left(a_{i j}\right)_{i, j=1}^{n}$ be an $n \times n$ matrix. Then

- $\quad A$ is said to be centrosymmetric if $A^{R}=A$.

- $\quad A$ is said to be persymmetric if $A^{R}=A^{\mathrm{T}}$. 
- $\quad A$ is said to be centrogonal if $A^{R}=A^{-1}$, provided $|A| \neq 0$.

- $\quad A$ is said to be skew-centrosymmetric if $A^{R}=-A$.

- $\quad A$ is said to be bisymmetric if $A^{R}=A=A^{\mathrm{T}}$.

Note that the bisymmetric matrix is both symmetric and centrosymmetric. It is also both symmetric and presymmetric.

\section{Some Properties of The Exchange and The Rotate Matrices}

Let us begin this section by giving some helpful results concerning the exchange and rotate matrices. For more details see [1,3,8,9,12-21].

The exchange matrix $J_{n}$ enjoys the following properties:

- $J=J^{\mathrm{T}}=J^{-1}$.

- $J_{n}^{k}= \begin{cases}I_{n}, & k \text { even } \\ J_{n}, & k \text { odd, }\end{cases}$

where $I_{n}$ is the identity matrix of order $n$.

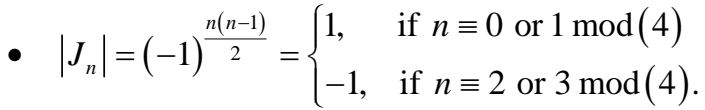

- The matrix product $J A$ is a version of the matrix $A$ that has been reflected in line at 0 degree to the horizontal measured counter-clockwise.

- The matrix product $A J$ is a version of the matrix A that has been reflected in line at 90 degrees to the horizontal measured counter-clockwise.

- The matrix product $J A J=A^{R}$ is a version of the matrix $A$ that has been rotated counter-clockwise or clockwise by 180 degrees.

Note that the exchange matrix $J$ is equal to the identity matrix $I$ but with the columns in reverse order. More precisely, $J=\left(e_{n}, e_{n-1}, \cdots, e_{1}\right)$ where $e_{i}=\left(\delta_{i 1}, \delta_{i 2}, \cdots, \delta_{n i}\right)$, $i=1,2, \cdots, n, \quad \delta_{i j}$ is the Kronecker symbol which is equal to 1 if $i=j$ and zero if $i \neq j$.

It is also worth mentioned that the matrix $J$ is sometimes called the counter-identity matrix or contra-identity matrix or per-identity matrix or the reflection matrix or the reversal matrix.

Exchange matrices are simple to construct in software platforms. For example, to construct $J_{5}$ in MAPLE, a single line of code can be used as follows:

$n:=5: J:=\operatorname{array}(1 . . n, 1 . . n$,sparse): for $i$ to $n$ do $J[i, n+$ $1-i]:=1$ od: $J \| n:=\operatorname{op}(J)$;

or $n:=5: J:=\operatorname{matrix}(n, n, 0)$ : for $i$ to $n$ do $J[i, \mathrm{n}+1$ i] : $=1$ od: $J \| n:=\operatorname{op}(J)$;

The rotate of $A, A^{R}$ of order $n$ satisfies:

- $\left(A^{R}\right)^{R}=A$.

- $\left(A^{R}\right)^{\mathrm{T}}=\left(A^{\mathrm{T}}\right)^{R}$.

- $\left(A^{-1}\right)^{R}=\left(A^{R}\right)^{-1}$, provided $A^{-1}$ exists.
- $(A B)^{R}=A^{R} B^{R}$.

- $(A \pm B)^{R}=\left(A^{R} \pm B^{R}\right)$.

- $\left|A^{R}\right|=|A|$.

- If $\operatorname{row}_{i}$ of $A$ is $\left(a_{i 1}, a_{i 2}, \cdots, a_{i, n-1}, a_{i n}\right)$, then $\operatorname{row}_{n+1-i}$ of $A^{R}$ is $\left(a_{i n}, a_{i, n-1}, \cdots, a_{i 2}, a_{i 1}\right)$. In other words, the centrosymmetric matrix $A$ is the same when read backwards as when read forwards.

\section{Centrosymmetric Determinants}

Centrosymmetric determinants take the form:

$$
\left|\begin{array}{cccc}
a_{1,1} & a_{1,2} & \cdots & a_{1, n} \\
a_{2,1} & a_{2,2} & \cdots & a_{2, n} \\
\vdots & \vdots & \vdots & \vdots \\
a_{2, n} & \cdots & a_{2,2} & a_{2,1} \\
a_{1, n} & \cdots & a_{1,2} & a_{1,1}
\end{array}\right|,
$$

in which

$$
\operatorname{row}_{i}=\left(a_{i 1}, a_{i 2}, \cdots, a_{i, n-1}, a_{i n}\right),
$$

and

$$
\operatorname{row}_{n+1-i}=\left(a_{i n}, a_{i, n-1}, \cdots, a_{i, 2}, a_{i 1}\right) \text {, }
$$

for each $i=1,2, \cdots,\left\lfloor\frac{n}{2}\right\rfloor$.

In particular, centrosymmetric tridiagonal determinants are of special importance. For convenience of the reader, we present some definitions, notations and properties associated with tridiagonal matrices.

A tridiagonal matrix $T=\left(a_{i j}\right)_{i, j=1}^{n}$ takes the form:

$$
T=\left[\begin{array}{ccccc}
d_{1} & a_{1} & 0 & \ldots & 0 \\
b_{2} & d_{2} & a_{2} & \ddots & \vdots \\
0 & \ddots & \ddots & \ddots & 0 \\
\vdots & \ddots & b_{n-1} & d_{n-1} & a_{n-1} \\
0 & \cdots & 0 & b_{n} & d_{n}
\end{array}\right] .
$$

These types of matrices frequently appear in many areas of science and engineering. For example in parallel computing, telecommunication system analysis and in solving differential equations using finite differences, see $[22,23]$. A general $n \times n$ tridiagonal matrix of the form (4) can be stored in $3 n$ memory locations, rather than $n^{2}$ memory locations for a full matrix, by using three vectors $\boldsymbol{a}=\left(a_{1}, a_{2}, \cdots, a_{n}\right), \quad \boldsymbol{b}=\left(b_{1}, b_{2}, \cdots, b_{n}\right), \quad$ and $\boldsymbol{d}=\left(d_{1}, d_{2}, \cdots, d_{n}\right)$ with $b_{1}=a_{n}=0$. This is always a good habit in computation in order to save memory space. To study tridiagonal matrices it is very convenient to introduce a vector $\boldsymbol{c}$ in the following way [24]:

$$
\boldsymbol{c}=\left(c_{1}, c_{2}, \cdots, c_{n}\right),
$$


where

$$
c_{1}=d_{1}, \quad c_{k}=d_{k}-\frac{b_{k} a_{k-1}}{c_{k-1}}, \quad k=2,3, \cdots, n .
$$

It is helpful to restate some important results concerning tridiagonal matrices of the form (4). For more details the interested reader may refer to [24-28].

Let $p, q, r$ and $s$ are positive integers such that $1 \leq p \leq q \leq n$ and $1 \leq r \leq s \leq n$.

Define the submatrix of $A$, denoted $A[p . . q, r . . s]$, of order $(q-p+1) \times(s-r+1)$ by

$$
A[p . . q, r . . s]=\left[\begin{array}{cccc}
a_{p, r} & a_{p, r+1} & \cdots & a_{p, s} \\
a_{p+1, r} & a_{p+1, r+1} & \cdots & a_{p+1, s} \\
\vdots & \vdots & \ddots & \vdots \\
a_{q, r} & a_{q, r+1} & \cdots & a_{q, s}
\end{array}\right] .
$$

In particular, let $F_{k}=A[1 . . k, 1 . . k], \quad 1 \leq k \leq n$, and $f_{k}=\left|F_{k}\right|$, the leading principal minor of $A$.

Theorem 3.1 [24]. Consider

$$
\begin{aligned}
& f_{1}=|T[1 . .1,1 . .1]|=\left|d_{1}\right|=d_{1}, \\
& f_{k}=|T[1 . . k, 1 . . k]|=\left|F_{k}\right|, k=2,3, \cdots, n .
\end{aligned}
$$

Then the determinants in (8) satisfy a three-term recurrence

$$
f_{k}=d_{k} f_{k-1}-b_{k} a_{k-1} f_{k-2}, k=1,2, \cdots, n,
$$

where the initial values for $f_{k}$ are $f_{0}=1$ and $f_{-1}=0$.

Lemma 3.2 [29]. If the $L U$ factorization of the matrix $T$ in (4) is possible, then we have

$$
|T|=\prod_{k=1}^{n} c_{k} .
$$

where $c_{1}, c_{2}, \cdots, c_{n}$ are given by (6). Meanwhile, the Doolittle $L U$ factorization [30] of $T$ is given by:

$$
\begin{aligned}
& T=L U \text {, where } \\
& L=\left[\begin{array}{ccccc}
1 & 0 & \cdots & \cdots & 0 \\
\frac{b_{2}}{c_{1}} & 1 & \ddots & & \vdots \\
0 & \ddots & \ddots & \ddots & 0 \\
\vdots & \ddots & \frac{b_{n-1}}{c_{n-2}} & 1 & 0 \\
0 & \ldots & 0 & \frac{b_{n}}{c_{n-1}} & 1
\end{array}\right], \\
& \text { and } U=\left[\begin{array}{ccccc}
c_{1} & a_{1} & 0 & \ldots & 0 \\
0 & c_{2} & a_{2} & \ddots & \vdots \\
\vdots & \ddots & \ddots & \ddots & 0 \\
\vdots & & \ddots & c_{n-1} & a_{n-1} \\
0 & \ldots & \ldots & 0 & c_{n}
\end{array}\right] .
\end{aligned}
$$

Lemma 3.3 [26]. If $c_{r} \neq 0, r=1,2, \cdots, n$, then

$$
f_{i}=\prod_{k=1}^{i} c_{k}, i=1,2, \cdots, n .
$$

Lemma 3.4 [26]. If $c_{i}=0,1 \leq i \leq n-1$, and either $a_{i}=0$ or $b_{i+1}=0$, then $T$ is a singular matrix.

Lemma 3.5 [24]. If $c_{i} \neq 0$ for each $i=1,2, \cdots, n-1$, then the three-term recurrence (9) reduces to the twoterm recurrence

$$
f_{i}=c_{i} f_{i-1}, \quad i=1,2, \cdots, n .
$$

Algorithm 3.1 (DETGTRI [31]).

The determinant of the matrix in (4) can be computed using the following symbolic algorithm.

INPUT: Order of the matrix $n$ and the components, $a_{i}, d_{i}, b_{i}, i=1,2, \cdots, n,\left(a_{n}=b_{1}=0\right)$.

OUTPUT: The determinant of the matrix $T$ in (4).

Step 1: Use (6) to compute the simplest forms of the $n$ components of the vector $\boldsymbol{c}$.

If $c_{i}=0$ for any $i \leq n$, set $c_{i}=t$ ( $t$ is just a symbolic name) and continue to compute $c_{i+1}, c_{i+2}, \cdots, c_{n}$ in terms of $t$ by using (6).

Step 2: The simplest rational form of the product $P(t)=\prod_{r=1}^{n} c_{r}$ (this product is a polynomial in $t$ ) evaluated at $t=0$ is equal to the determinant of the matrix $T$ in (4), i.e., $|T|=P(0)$.

The cost of the DETGTRI algorithm is $O(n)$. The algorithm is easy to implement in all Computer Algebra Systems (CAS) such as MACSYMA, MATHEMATICA and MAPLE.

Lemma 3.6. Consider the tridiagonal matrix $T_{n}$ given by:

$$
T_{n}=\left[\begin{array}{ccccc}
d_{1} & a_{1} & 0 & \ldots & 0 \\
b_{2} & d_{2} & a_{2} & \ddots & \vdots \\
0 & \ddots & \ddots & \ddots & 0 \\
\vdots & \ddots & b_{n-1} & d_{n-1} & a_{n-1} \\
0 & \cdots & 0 & b_{n} & d_{n}
\end{array}\right] .
$$

Let $S_{n}, U_{n}$ and $V_{n}$ be $n \times n$ matrices defined respectively as follows:

$$
\begin{aligned}
& S_{n}=\left.T_{n}\right|_{d_{n} \rightarrow d_{n} \pm k}, \quad U_{n}=\left.T_{n}\right|_{a_{n-1} \rightarrow k a_{n-1}} \\
& \text { and } V_{n}=\left.T_{n}\right|_{b_{n} \rightarrow k b_{n}},
\end{aligned}
$$

where $k$ is a scalar quantity. Then by applying the DETGTRI algorithm, we see that:

$$
\begin{aligned}
& \left|S_{n}\right|=\left|T_{n}\right| \pm k\left|T_{n-1}\right|, \\
& \left|U_{n}\right|=\left|V_{n}\right|=\left|T_{n}\right|-(k-1) b_{n} a_{n-1}\left|T_{n-2}\right|,
\end{aligned}
$$

having used (12) and (13).

Let $A=\left(a_{i j}\right)_{i, j=1}^{n}$ is an $n \times n$ centrosymmetric matrix, then the three following facts are useful when we 
deal with such matrices:

Fact (1): If $\operatorname{row}_{i}=\left(a_{i 1}, a_{i 2}, \cdots, a_{i, n-1}, a_{i n}\right)$, then

$$
\operatorname{row}_{n+1-i}=\left(a_{i n}, a_{i, n-1}, \cdots, a_{i 2}, a_{i 1}\right) \text {. }
$$

In other words, the centrosymmetric matrix $A$ is the same when read backwards as when read forwards.

Fact (2): If $n=2 m$ is positive even number, then

$$
Q_{1}=\frac{1}{\sqrt{2}}\left[\begin{array}{cc}
I_{m} & I_{m} \\
J_{m} & -J_{m}
\end{array}\right]
$$

is orthogonal.

Fact (3): If $n=2 m+1$ is positive odd number then

$$
Q_{2}=\frac{1}{\sqrt{2}}\left[\begin{array}{ccc}
I_{m} & 0 & I_{m} \\
0 & \sqrt{2} & 0 \\
J_{m} & 0 & -J_{m}
\end{array}\right],
$$

is orthogonal.

Armed with the above facts, we may formulate the following result whose proof will be omitted.

Theorem 3.7. Let $R=\left(r_{i j}\right)_{i, j=1}^{n}$ be an $n \times n$ of even order say $n=2 m$, then $R$ can be written in the form:

$$
R=\left[\begin{array}{ll}
A & J B J \\
B & J A J
\end{array}\right]=Q_{1}\left[\begin{array}{ll}
A+J B & \\
& A-J B
\end{array}\right] Q_{1}^{\mathrm{T}},
$$

where $A, B \in \mathbb{R}^{m \times m}$.

The determinant of the matrix $R$ in (18) is given by:

$$
|R|=|A+J B||A-J B| .
$$

$$
T_{n}=T_{2 m}=\left[\begin{array}{ccccc|ccccc}
d_{1} & a_{1} & 0 & \cdots & 0 & 0 & \cdots & & \cdots & 0 \\
b_{2} & d_{2} & a_{2} & \ddots & \vdots & \vdots & & & & \vdots \\
0 & \ddots & \ddots & \ddots & 0 & & & & & \\
\vdots & \ddots & b_{m-1} & d_{m-1} & a_{m-1} & 0 & & & & \vdots \\
0 & \cdots & 0 & b_{m} & d_{m} & k & 0 & & \cdots & 0 \\
\hline 0 & \cdots & & 0 & k & d_{m} & b_{m} & 0 & \cdots & 0 \\
\vdots & & & & 0 & a_{m-1} & d_{m-1} & b_{m-1} & \ddots & \vdots \\
& & & & & 0 & \ddots & \ddots & \ddots & 0 \\
\vdots & & & & \vdots & \vdots & \ddots & a_{2} & d_{2} & b_{2} \\
0 & \cdots & & \cdots & 0 & 0 & \cdots & 0 & a_{1} & d_{1}
\end{array}\right] .
$$

Applying Theorem 3.7, we have $\left|T_{n}\right|=\left|T_{2 m}\right|=|L||M|$, where

$$
L=A+J B=\left[\begin{array}{ccccc}
d_{1} & a_{1} & 0 & \cdots & 0 \\
b_{2} & d_{2} & a_{2} & \ddots & \vdots \\
0 & \ddots & \ddots & \ddots & 0 \\
\vdots & \ddots & b_{m-1} & d_{m-1} & a_{m-1} \\
0 & \cdots & 0 & b_{m} & d_{m}+k
\end{array}\right]
$$

and 


$$
M=A-J B=\left[\begin{array}{ccccc}
d_{1} & a_{1} & 0 & \cdots & 0 \\
b_{2} & d_{2} & a_{2} & \ddots & \vdots \\
0 & \ddots & \ddots & \ddots & 0 \\
\vdots & \ddots & b_{m-1} & d_{m-1} & a_{m-1} \\
0 & \cdots & 0 & b_{m} & d_{m}-k
\end{array}\right] .
$$

By using Lemma 3.6, we obtain

$$
|L|=\left|T_{m}\right|+k\left|T_{m-1}\right|
$$

and

$$
|M|=\left|T_{m}\right|-k\left|T_{m-1}\right| .
$$

Therefore we get

$$
\begin{aligned}
\left|T_{n}\right| & =\left|T_{2 m}\right|=|L||M| \\
& =\left|T_{m}\right|^{2}-k^{2}\left|T_{m-1}\right|^{2} .
\end{aligned}
$$

Case (2): $n=2 m+1$. In this case, the centrosymmetric tridiagonal matrix takes the form:

$$
T_{n}=T_{2 m+1}=\left[\begin{array}{ccccc|c|ccccc}
d_{1} & a_{1} & 0 & \cdots & 0 & 0 & 0 & \cdots & & \cdots & 0 \\
b_{2} & d_{2} & a_{2} & \ddots & \vdots & \vdots & \vdots & & & & \vdots \\
0 & \ddots & \ddots & \ddots & 0 & \vdots & & & & & \\
\vdots & \ddots & b_{m-1} & d_{m-1} & a_{m-1} & 0 & \vdots & & & & \vdots \\
0 & \cdots & 0 & b_{m} & d_{m} & a_{m} & 0 & \cdots & & \cdots & 0 \\
\hline 0 & \cdots & \cdots & 0 & b_{m+1} & d_{m+1} & b_{m+1} & 0 & \cdots & & 0 \\
\hline 0 & \cdots & & \cdots & 0 & a_{m} & d_{m} & b_{m} & 0 & \cdots & 0 \\
\vdots & & & & \vdots & 0 & a_{m-1} & d_{m-1} & b_{m-1} & \ddots & \vdots \\
\vdots & & & & & \vdots & 0 & \ddots & \ddots & \ddots & 0 \\
0 & \cdots & & \cdots & 0 & 0 & 0 & \cdots & 0 & a_{1} & d_{1}
\end{array}\right] .
$$

Applying Theorem 3.7, we obtain $\left|T_{n}\right|=\left|T_{2 m+1}\right|=|L||M|$, here

$$
L=A-J B=A=T_{m}=\left[\begin{array}{ccccc}
d_{1} & a_{1} & 0 & \cdots & 0 \\
b_{2} & d_{2} & a_{2} & \ddots & \vdots \\
0 & \ddots & \ddots & \ddots & 0 \\
\vdots & \ddots & b_{m-1} & d_{m-1} & a_{m-1} \\
0 & \cdots & 0 & b_{m} & d_{m}
\end{array}\right]
$$

and

$$
M=\left[\begin{array}{cc}
A+J B & \sqrt{2} \boldsymbol{v} \\
\sqrt{2} \boldsymbol{u}^{\mathrm{T}} & q
\end{array}\right]=\left[\begin{array}{ccccc}
d_{1} & a_{1} & 0 & \cdots & 0 \\
b_{2} & d_{2} & a_{2} & \ddots & \vdots \\
0 & \ddots & \ddots & \ddots & 0 \\
\vdots & \ddots & b_{m} & d_{m} & \sqrt{2} a_{m} \\
0 & \cdots & 0 & \sqrt{2} b_{m+1} & d_{m+1}
\end{array}\right] .
$$

By using Lemma 3.6, we obtain $|L|=\left|T_{m}\right|$ and

$$
|M|=\left|\begin{array}{cc}
A+J B & \sqrt{2} \boldsymbol{v} \\
\sqrt{2} \boldsymbol{u}^{\mathrm{T}} & q
\end{array}\right|=\left|\begin{array}{ccccc}
d_{1} & a_{1} & 0 & \cdots & 0 \\
b_{2} & d_{2} & a_{2} & \ddots & \vdots \\
0 & \ddots & \ddots & \ddots & 0 \\
\vdots & \ddots & b_{m} & d_{m} & \sqrt{2} a_{m} \\
0 & \cdots & 0 & \sqrt{2} b_{m+1} & d_{m+1}
\end{array}\right| .
$$

By using the DETGTRI algorithm [31] together with (12) and (13), then we have 


$$
\begin{aligned}
|M| & =\left|\begin{array}{ccccc}
d_{1} & a_{1} & 0 & \cdots & 0 \\
b_{2} & d_{2} & a_{2} & \ddots & \vdots \\
0 & \ddots & \ddots & \ddots & 0 \\
\vdots & \ddots & b_{m} & d_{m} & 2 a_{m} \\
0 & \cdots & 0 & b_{m+1} & d_{m+1}
\end{array}\right|=\left|\begin{array}{ccccc}
d_{1} & a_{1} & 0 & \cdots & 0 \\
b_{2} & d_{2} & a_{2} & \ddots & \vdots \\
0 & \ddots & \ddots & \ddots & 0 \\
\vdots & \ddots & b_{m} & d_{m} & a_{m} \\
0 & \cdots & 0 & 2 b_{m+1} & d_{m+1}
\end{array}\right| \\
& =\left(c_{1} c_{2} \cdots c_{m}\right) \alpha_{m+1}=\left|T_{m}\right| \alpha_{m+1}=\left|T_{m}\right|\left(d_{m+1}-2 \frac{b_{m+1} a_{m}}{c_{m}}\right)=\left|T_{m}\right|\left(c_{m+1}-\frac{b_{m+1} a_{m}}{c_{m}}\right) \\
& =c_{m+1}\left|T_{m}\right|-\frac{\left|T_{m}\right|}{c_{m}} b_{m+1} a_{m}=\left|T_{m+1}\right|-\frac{\left|T_{m}\right|}{c_{m}} b_{m+1} a_{m}=\left|T_{m+1}\right|-b_{m+1} a_{m}\left|T_{m-1}\right| .
\end{aligned}
$$

Therefore

$$
\begin{aligned}
\left|T_{n}\right| & =\left|T_{2 m+1}\right| \\
& =\left|T_{m}\right|\left(\left|T_{m+1}\right|-b_{m+1} a_{m}\left|T_{m-1}\right|\right) .
\end{aligned}
$$

From (23) and (25), we see that in order to compute the determinant of a centrosymmetric tridiagonal matrix of order $n$, then all we need is to compute $c_{1}, c_{2}, \cdots, c_{m}$ if $n=2 m$ and $c_{1}, c_{2}, \cdots, c_{m+1}$ if $n=2 m+1$.

\section{Algorithms for Solving Centrosymmetric Linear Systems}

Solving linear systems practically dominates scientific computing. In the present section, we focus on solving linear systems of centrosymmetric type. Two cases will be considered:

Case (i): $n=2 m$. For this case we are going to construct an algorithm for solving centrosymmetric linear systems of the form:

$$
\left[\begin{array}{cccccccc}
a_{11} & a_{12} & \cdots & a_{1, m} & a_{1, m+1} & \cdots & a_{1,2 m-1} & a_{1,2 m} \\
a_{21} & a_{22} & \cdots & a_{2, m} & a_{2, m+1} & \cdots & a_{2,2 m-1} & a_{2,2 m} \\
\vdots & \vdots & \ddots & \vdots & \vdots & \ddots & \vdots & \vdots \\
a_{m 1} & a_{m 2} & \cdots & a_{m, m} & a_{m, m+1} & \cdots & a_{m, 2 m-1} & a_{m, 2 m} \\
a_{m, 2 m} & a_{m, 2 m-1} & \cdots & a_{m, m+1} & a_{m, m} & \cdots & a_{m, 2} & a_{m, 1} \\
\vdots & \vdots & \ddots & \vdots & \vdots & \ddots & \vdots & \vdots \\
a_{2,2 m} & a_{2,2 m-1} & \cdots & a_{2, m+1} & a_{2, m} & \cdots & a_{22} & a_{21} \\
a_{1,2 m} & a_{1,2 m-1} & \cdots & a_{1, m+1} & a_{1, m} & \cdots & a_{12} & a_{11}
\end{array}\right]\left[\begin{array}{c}
x_{1} \\
x_{2} \\
\vdots \\
x_{m} \\
\vdots \\
x_{2 m-1} \\
x_{2 m}
\end{array}\right]=\left[\begin{array}{c}
b_{1} \\
b_{2} \\
\vdots \\
b_{m} \\
\vdots \\
b_{2 m-1} \\
b_{2 m}
\end{array}\right] .
$$

Block multiplication is particularly useful when there are patterns in the matrices to be multiplied. Therefore it is convenient to rewrite (26) in the partitioned form

$$
\left[\begin{array}{c|c}
A & J B J \\
\hline B & J A J
\end{array}\right]\left[\begin{array}{l}
X_{1} \\
\hline X_{2}
\end{array}\right]=\left[\frac{B_{1}}{B_{2}}\right],
$$

where

$$
\begin{aligned}
& A=\left[\begin{array}{cccc}
a_{11} & a_{12} & \cdots & a_{1, m} \\
a_{21} & a_{22} & \cdots & a_{2, m} \\
\vdots & \vdots & \ddots & \vdots \\
a_{m 1} & a_{m 2} & \cdots & a_{m, m}
\end{array}\right] \quad B=\left[\begin{array}{cccc}
a_{m, 2 m} & a_{m, 2 m-1} & \cdots & a_{m, m+1} \\
\vdots & \vdots & \ddots & \vdots \\
a_{2,2 m} & a_{2,2 m-1} & \cdots & a_{2, m+1} \\
a_{1,2 m} & a_{1,2 m-1} & \cdots & a_{1, m+1}
\end{array}\right], \\
& X_{1}=\left[x_{1}, x_{2}, \cdots, x_{m}\right]^{\mathrm{T}}, X_{2}=\left[x_{m+1}, x_{m+2}, \cdots, x_{2 m}\right]^{\mathrm{T}}, \\
& B_{1}=\left[b_{1}, b_{2}, \cdots, b_{m}\right]^{\mathrm{T}} \text { and } B_{2}=\left[b_{m+1}, b_{m+2}, \cdots, b_{2 m}\right]^{\mathrm{T}} .
\end{aligned}
$$

The system in (26) can also be written in matrix form as follows:

$$
R \boldsymbol{x}=\boldsymbol{b}
$$

where $R=\left(a_{i j}\right)_{i, j=1}^{2 m}$ is the coefficient matrix of the system (26), $\boldsymbol{x}=\left[x_{1}, x_{2}, \cdots, x_{2 m}\right]^{\mathrm{T}}$ and $\boldsymbol{b}=\left[b_{1}, b_{2}, \cdots, b_{2 m}\right]^{\mathrm{T}}$ 
is the constant vector.

Algorithm 4.1. An algorithm for solving centrosymmetric linear system of even order.

To solve the linear system of the form (26), we may proceed as follows:

INPUT: The entries of the coefficient matrix $R$ and the constant vector $\boldsymbol{b}$ in (28).

OUTPUT: Solution vector $\boldsymbol{x}=\left[x_{1}, x_{2}, \cdots, x_{2 m}\right]^{\mathrm{T}}$.

Step 1: Construct the $m \times m$ matrices $P, Q$ and the m-vectors $\hat{\boldsymbol{b}}$ and $\tilde{\boldsymbol{b}}$ as follows:

$$
\begin{aligned}
& P=A+J B=\left[a_{i j}+a_{i, 2 m+1-j}\right]_{i, j=1}^{m}, \\
& Q=A-J B=\left[a_{i j}-a_{i, 2 m+1-j}\right]_{i, j=1}^{m}, \\
& \hat{\boldsymbol{b}}=\left[b_{1}+b_{2 m}, b_{2}+b_{2 m-1}, \cdots, b_{m}+b_{m+1}\right]^{\mathrm{T}}
\end{aligned}
$$

and

$$
\tilde{\boldsymbol{b}}=\left[b_{1}-b_{2 m}, b_{2}-b_{2 m-1}, \cdots, b_{m}-b_{m+1}\right]^{\mathrm{T}} .
$$

Step 2: Compute $|R|=|P||Q|$. If $|R|=0$ then Exiterror('No solutions') end if.

Step 3: Solve the two linear systems:

$\boldsymbol{P y}=\hat{\boldsymbol{b}}$, and $\mathbf{Q z}=\tilde{\boldsymbol{b}}$, for $\boldsymbol{y}=\left[y_{1}, y_{2}, \cdots, y_{m}\right]^{\mathrm{T}}$ and $\mathbf{z}=\left[z_{1}, z_{2}, \cdots, z_{m}\right]^{\mathrm{T}}$ respectively.

Step 4: The solution vector $\boldsymbol{x}=\left[x_{1}, x_{2}, \cdots, x_{2 m}\right]^{\mathrm{T}}$ is given by

$$
x_{i}= \begin{cases}\frac{1}{2}\left(y_{i}+z_{i}\right) & \text { if } i=1,2, \cdots, m, \\ \frac{1}{2}\left(y_{2 m+1-i}-z_{2 m+1-i}\right) & \text { if } i=m+1, m+2, \cdots, 2 m .\end{cases}
$$

The Algorithm 4.1, will be refereed to as CENTROSYMM-I algorithm.

Concerning the computational cost of the CENTRO-
SYMM-I algorithm:

- The time complexity of Step $\mathbf{1}$ is

$$
\begin{aligned}
& m^{2}+m^{2}+m+m \\
& =2 m^{2}+2 m=2 \times\left(\frac{n}{2}\right)^{2}+2 \times \frac{n}{2} \\
& =\frac{1}{2} n^{2}+n=\frac{1}{2} n^{2}+O(n)
\end{aligned}
$$

$\left(\frac{1}{2} n^{2}+n\right.$ additions/subtractions and no multiplications/divisions).

- The time complexity of Step 3 depends on the solvers of the two linear systems. For example, tridiagonal linear systems can be solved in linear time (see [25]).

- The time complexity of Step 4 is $O(n)$ ( $n$ additions/subtractions and $n$ multiplications).

Step 3 is the step that leads to the reduction of the time complexity, because instead of solving a linear system of $n$ equations, we end up with two linear systems half the size of the original one. If the original system is solved with Gaussian elimination (GE) method, then the time complexity will be $\frac{2}{3} n^{3}+O\left(n^{2}\right)$. But, if GE method is used to solve the two systems in the third step, then the time complexity of our algorithm will be

$$
\frac{4}{3} m^{3}+O\left(m^{2}\right)=\frac{1}{6} n^{3}+O\left(n^{2}\right),
$$

which is a significant reduction. If a method more efficient than the GE method is used, then the time complexity of our algorithm will be less (see also [16]).

Case (ii): $n=2 m+1$. In this case the linear system to be considered has the form:

$$
\left[\begin{array}{ccccccccc}
a_{11} & a_{12} & \cdots & a_{1, m} & a_{1, m+1} & a_{1, m+2} & \cdots & a_{1,2 m} & a_{1,2 m+1} \\
a_{21} & a_{22} & \cdots & a_{2, m} & a_{2, m+1} & a_{2, m+2} & \cdots & a_{2,2 m} & a_{2,2 m+1} \\
\vdots & \vdots & \ddots & \vdots & \vdots & \vdots & \ddots & \vdots & \vdots \\
a_{m 1} & a_{m 2} & \cdots & a_{m, m} & a_{m, m+1} & a_{m, m+2} & \cdots & a_{m, 2 m} & a_{m, 2 m+1} \\
a_{m+1,1} & a_{m+1,2} & \cdots & a_{m+1, m} & a_{m+1, m+1} & a_{m+1, m} & \cdots & a_{m+1,2} & a_{m+1,1} \\
a_{m, 2 m+1} & a_{m, 2 m} & \cdots & a_{m, m+2} & a_{m, m+1} & a_{m, m} & \cdots & a_{m, 2} & a_{m, 1} \\
\vdots & \vdots & \ddots & \vdots & \vdots & \vdots & \ddots & \vdots & \vdots \\
a_{2,2 m+1} & a_{2,2 m} & \cdots & a_{2, m+2} & a_{2, m+1} & a_{2, m} & \cdots & a_{22} & a_{21} \\
a_{1,2 m+1} & a_{1,2 m} & \cdots & a_{1, m+2} & a_{1, m+1} & a_{1, m} & \cdots & a_{12} & a_{11}
\end{array}\right]\left[\begin{array}{c}
x_{1} \\
x_{2} \\
\vdots \\
x_{m+1} \\
\vdots \\
x_{2 m} \\
x_{2 m+1}
\end{array}\right]=\left[\begin{array}{c}
b_{1} \\
b_{2} \\
\vdots \\
b_{m+1} \\
\vdots \\
b_{2 m} \\
b_{2 m+1}
\end{array}\right],
$$

or equivalently,

$$
\left[\begin{array}{c|c|c}
A & \boldsymbol{v} & J B J \\
\hline \mathbf{u}^{\mathrm{T}} & q & \boldsymbol{u}^{\mathrm{T}} J \\
\hline B & J \boldsymbol{v} & J A J
\end{array}\right]\left[\begin{array}{c}
\frac{X_{1}}{x_{m+1}} \\
\hline X_{2}
\end{array}\right]=\left[\begin{array}{c}
\frac{B_{1}}{b_{m+1}} \\
B_{2}
\end{array}\right],
$$


where

$$
\begin{aligned}
& A=\left[\begin{array}{cccc}
a_{11} & a_{12} & \cdots & a_{1, m} \\
a_{21} & a_{22} & \cdots & a_{2, m} \\
\vdots & \vdots & \ddots & \vdots \\
a_{m 1} & a_{m 2} & \cdots & a_{m, m}
\end{array}\right] \quad B=\left[\begin{array}{cccc}
a_{m, 2 m+1} & a_{m, 2 m} & \cdots & a_{m, m+2} \\
\vdots & \vdots & \ddots & \vdots \\
a_{2,2 m+1} & a_{2,2 m} & \cdots & a_{2, m+2} \\
a_{1,2 m+1} & a_{1,2 m} & \cdots & a_{1, m+2}
\end{array}\right], \\
& \boldsymbol{v}=\left[a_{1, m+1}, a_{2, m+1}, \cdots, a_{m, m+1}\right]^{\mathrm{T}}, \quad \boldsymbol{u}=\left[a_{m+1,1}, a_{m+1,2}, \cdots, a_{m+1, m}\right]^{\mathrm{T}}, \quad q=\left[a_{m+1, m+1}\right], \\
& X_{1}=\left[x_{1}, x_{2}, \cdots, x_{m}\right]^{\mathrm{T}}, \quad X_{2}=\left[x_{m+2}, x_{m+3}, \cdots, x_{2 m+1}\right]^{\mathrm{T}}, \\
& B_{1}=\left[b_{1}, b_{2}, \cdots, b_{m}\right]^{\mathrm{T}} \text { and } B_{2}=\left[b_{m+2}, b_{m+3}, \cdots, b_{2 m+1}\right]^{\mathrm{T}} .
\end{aligned}
$$

The linear system (30) can also be written as:

$$
R \boldsymbol{x}=\boldsymbol{b},
$$

where $R=\left(a_{i j}\right)_{i, j=1}^{2 m+1}$ is the coefficient matrix of the system (29),

$$
\boldsymbol{x}=\left[x_{1}, x_{2}, \cdots, x_{2 m+1}\right]^{\mathrm{T}} \text { and } \boldsymbol{b}=\left[b_{1}, b_{2}, \cdots, b_{2 m+1}\right]^{\mathrm{T}}
$$

is the constant vector.

Algorithm 4.2. An algorithm for solving centrosym- metric linear system of odd order

To solve the linear system of the form (29), we may proceed as follows:

INPUT: The entries of the coefficient matrix $R$ and the constant vector $\boldsymbol{b}$ in (31).

OUTPUT: Solution vector $\boldsymbol{x}=\left[x_{1}, x_{2}, \cdots, x_{2 m+1}\right]^{\mathrm{T}}$.

Step 1: Construct the matrices $P, Q$ of orders $m+1$ and $m$ respectively and the vectors $\hat{\boldsymbol{b}}$ and $\tilde{\boldsymbol{b}}$ of dimensions $m+1$ and $m$ respectively as follows:

$$
P=\left[\begin{array}{cc}
A+J B & 2 \boldsymbol{v} \\
\boldsymbol{u}^{\mathrm{T}} & q
\end{array}\right]=\left[\begin{array}{ccccc}
a_{1,1}+a_{1,2 m+1} & a_{1,2}+a_{1,2 m} & \cdots & a_{1, m}+a_{1, m+2} & 2 a_{1, m+1} \\
a_{2,1}+a_{2,2 m+1} & a_{2,2}+a_{2,2 m} & \cdots & a_{2, m}+a_{2, m+2} & 2 a_{2, m+1} \\
\vdots & \vdots & \ddots & \vdots & \vdots \\
a_{m, 1}+a_{m, 2 m+1} & a_{m, 2}+a_{m, 2 m} & \cdots & a_{m, m}+a_{m, m+2} & 2 a_{m, m+1} \\
a_{m+1,1} & a_{m+1,2} & \cdots & a_{m+1, m} & a_{m+1, m+1}
\end{array}\right],
$$$$
Q=A-J B=\left[a_{i j}-a_{i, 2 m+2-j}\right]_{i, j=1}^{m},
$$$$
\hat{\boldsymbol{b}}=\left[b_{1}+b_{2 m+1}, b_{2}+b_{2 m}, \cdots, b_{m}+b_{m+2}, b_{m+1}\right]^{\mathrm{T}}
$$

and

$$
\tilde{\boldsymbol{b}}=\left[b_{1}-b_{2 m+1}, b_{2}-b_{2 m}, \cdots, b_{m}-b_{m+2}\right]^{\mathrm{T}} .
$$

Step 2: Compute $|R|=|P||Q|$, If $|R|=0$ then Ex-

$$
x_{i}= \begin{cases}\frac{1}{2}\left(y_{i}+z_{i}\right) & \text { if } i=1,2, \cdots, m, \\ y_{m+1} & \text { if } i=m+1, \\ \frac{1}{2}\left(y_{2 m+2-i}-z_{2 m+2-i}\right) & \text { if } i=m+2, m+3, \cdots, 2 m+1 .\end{cases}
$$

The Algorithm will be refereed to as CENTROSYMM-II algorithm.

Concerning the computational cost of the CENTRO-
iterror("No solutions") end if.

Step 3: Solve the two linear systems $P \boldsymbol{y}=\hat{\boldsymbol{b}}$, and $Q \mathbf{z}=\tilde{\boldsymbol{b}}$, for

$$
\boldsymbol{y}=\left[y_{1}, y_{2}, \cdots, y_{m}, y_{m+1}\right]^{\mathrm{T}} \text { and } \boldsymbol{z}=\left[z_{1}, z_{2}, \cdots, z_{m}\right]^{\mathrm{T}}
$$

respectively.

Step 4: The solution vector $\boldsymbol{x}=\left[x_{1}, x_{2}, \cdots, x_{2 m+1}\right]^{\mathrm{T}}$ is given by

SYMM-II algorithm:

- The time complexity of Step 1 is $\frac{1}{2} n^{2}+O(n)$. 
- The time complexity of Step 3 depends on the solvers of the two linear systems.

- The time complexity of Step 4 is $O(n)$ (see also [16]).

It may be convenient to finish this section by giving the following result, whose proof will be omitted.

Theorem 4.1. Let $A=\left(a_{i j}\right)_{i, j=1}^{n}$ be a non-singular centrosymmetric square matrix of order $n$. Consider the four linear systems of centrosymmetric type:

$$
\begin{aligned}
& A \boldsymbol{x}=b, \\
& A J \boldsymbol{x}=b, \\
& J A \boldsymbol{x}=b
\end{aligned}
$$

and

$$
A^{R} \boldsymbol{x}=b .
$$

Then the two linear systems (32) and (35) are equivalent. The same is true for the linear systems (33) and (34). Moreover, if the common solution of the systems (32) and (35) is $\boldsymbol{x}=\left(k_{1}, k_{2}, \cdots, k_{n}\right)^{\mathrm{T}}$, then the common solution of the systems (33) and (34) is $\boldsymbol{x}=\left(k_{n}, k_{n-1}, \cdots, k_{1}\right)^{\mathrm{T}}$.

\section{Computer Program}

In this section, we are going to introduce a MAPLE procedure for solving centrosymmetric linear systems (26) and (29). This procedure is based on the CENTROSYMM-I and CENTROSYMM-II Algorithms.

> restart:

centrosymm:=proc(R::array,f::vector,n::posint) local i, r,m,f1,f2,A,Jm,J,H,y,x,B,Y,Z,X:

global xsoln,detR,detP,detQ,P,Q; sparse):

$\mathrm{X}:=\operatorname{vector}(\mathrm{n}): \mathrm{m}:=$ floor(n/2): J:=array(1..m, $1 . . \mathrm{m}$,

for $\mathrm{i}$ to $\mathrm{m}$ do $\mathrm{J}[\mathrm{m}+1-\mathrm{i}, \mathrm{i}]:=1$ od:

A:= linalg[submatrix] (R,1..m,1..m):

if $n=2 * m$ then

\# Case(1): $n$ is even \#

\# Step 1 in CENTROSYMM-I Algorithm.

$\mathrm{B}:=$ linalg[submatrix $](\mathrm{R}, \mathrm{m}+1 . . \mathrm{n}, 1 . . \mathrm{m})$ :

$\mathrm{P}:=\operatorname{evalm}(\mathrm{A}+\operatorname{evalm}(\mathrm{J} \& * \mathrm{~B}))$ :

$\mathrm{Q}:=\operatorname{evalm}(\mathrm{A}$ - evalm(J\&*B)):

\# Step 2 in CENTROSYMM-I Algorithm.

$\operatorname{detP}:=\operatorname{linalg}[\operatorname{det}](\mathrm{P}): \operatorname{det} \mathrm{Q}:=\operatorname{linalg}[\operatorname{det}](\mathrm{Q})$ :

$\operatorname{det} R:=\operatorname{det} P * \operatorname{det} Q$ :

if $\operatorname{detR}=0$ then ERROR("Singular Matrix !!!! ")

fi;

\# Step 3 in CENTROSYMM-I Algorithm.

$\mathrm{f} 1:=\operatorname{array}(1 . . \mathrm{m})$ : f2:=array(1..m):

for $i$ to $m$ do

f1[i]:=f[i] $+f[2 * m+1-i]$; od;

f2[i]:=f[i]-f[2*m+1-i];

$\mathrm{Y}:=\operatorname{array}(1 . . \mathrm{m}): \mathrm{Z}:=\operatorname{array}(1 . . \mathrm{m})$ :

$\mathrm{Y}:=$ linalg[linsolve](P,f1): $\mathrm{Z}:=$ linalg[linsolve]

$(\mathrm{Q}, \mathrm{f} 2)$ :

\# Step 4 in CENTROSYMM-I Algorithm.

for $\mathrm{i}$ to $\mathrm{m}$ do

$\mathrm{X}[\mathrm{i}]:=1 / 2 *(\mathrm{Y}[\mathrm{i}]+\mathrm{Z}[\mathrm{i}])$;

od;

for i from $m+1$ to $n$ do

$X[i]:=1 / 2 *(Y[n+1-i]-Z[n+1-i])$;

od;

xsoln:=simplify([seq(X[r],r=1..n)]):

else

\# Case(2): $\mathrm{n}$ is odd \#

\# Step 1 in CENTROSYMM-II Algorithm.

$\mathrm{B}:=\operatorname{linalg}[$ submatrix $](\mathrm{R}, \mathrm{m}+2 . . \mathrm{n}, 1 . . \mathrm{m})$ :

$\mathrm{H}:=\operatorname{evalm}(\mathrm{A}+\operatorname{evalm}(\mathrm{J} \& * \mathrm{~B}))$ :

$\mathrm{y}:=\operatorname{linalg}[$ submatrix $](\mathrm{R}, 1 . . \mathrm{m}, \mathrm{m}+1 . . \mathrm{m}+1)$ :

$\mathrm{x}:=\operatorname{linalg}[$ submatrix $](\mathrm{R}, \mathrm{m}+1 . . \mathrm{m}+1,1 . . \mathrm{m})$ :

$\mathrm{P}:=\operatorname{linalg}[$ blockmatrix] $(2,2,[\mathrm{H}, 2 * \mathrm{y}, \mathrm{x},[2]]): \mathrm{op}(\mathrm{P})$;

Q:=evalm( A - evalm(J\&*B)):

\# Step 2 in CENTROSYMM-II Algorithm.

$\operatorname{detP}:=\operatorname{linalg}[\operatorname{det}](\mathrm{P}): \operatorname{det} \mathrm{Q}:=\operatorname{linalg}[\operatorname{det}](\mathrm{Q}): \operatorname{det} \mathrm{R}:=$ $\operatorname{det} \mathrm{P} * \operatorname{det} \mathrm{Q}$ :

if $\operatorname{detR}=0$ then ERROR("Singular Matrix !!!!")

fi;

\# Step 3 in CENTROSYMM-II Algorithm.

$\mathrm{f} 1:=\operatorname{array}(1 . . \mathrm{m}+1)$ : f2:=array $(1 . . \mathrm{m})$ :

for $i$ to $m$ do

$\mathrm{f} 1[\mathrm{i}]:=\mathrm{f}[\mathrm{i}]+\mathrm{f}[2 * \mathrm{~m}+2-\mathrm{i}]$;

$\mathrm{f} 2[\mathrm{i}]:=\mathrm{f}[\mathrm{i}]-\mathrm{f}[2 * \mathrm{~m}+2-\mathrm{i}]$;

od;

f1[m+1]:=f[m+1];

$\mathrm{Y}:=\operatorname{array}(1 . . \mathrm{m}+1)$ :

$\mathrm{Y}:=\quad$ linalg[linsolve](P,f1): $\quad \mathrm{Z}:=\operatorname{linalg[linsolve]}$

(Q,f2):

\# Step 4 in CENTROSYMM-II Algorithm.

for $\mathrm{i}$ to $\mathrm{m}$ do

od;

$X[i]:=1 / 2 *(Y[i]+Z[i])$;

$\mathrm{X}[\mathrm{m}+1]:=\mathrm{Y}[\mathrm{m}+1]$;

for i from $m+2$ to $n$ do

$$
X[i]:=1 / 2 *(Y[n+1-i]-Z[n+1-i]) \text {; }
$$

od;

fi:

xsoln:=simplify([seq(X[r],r=1..n)]);

end proc:

\section{Illustrative Examples}

All results in this section are obtained with the help of the MAPLE procedure centrosymm.

Example 6.1. Solve the centrosymmetric linear system 


$$
\left[\begin{array}{cccccccccc}
3 & -1 & 1 & 0 & 4 & 1 & -3 & 2 & -3 & 1 \\
2 & -1 & -3 & 4 & -2 & -1 & -1 & 0 & 3 & 2 \\
1 & 0 & 2 & -2 & 3 & 3 & 2 & 1 & 3 & 1 \\
3 & 3 & -3 & 4 & -1 & 4 & -2 & 3 & -1 & 1 \\
1 & 1 & 2 & -1 & 3 & 2 & 1 & -1 & 3 & 0 \\
0 & 3 & -1 & 1 & 2 & 3 & -1 & 2 & 1 & 1 \\
1 & -1 & 3 & -2 & 4 & -1 & 4 & -3 & 3 & 3 \\
1 & 3 & 1 & 2 & 3 & 3 & -2 & 2 & 0 & 1 \\
2 & 3 & 0 & -1 & -1 & -2 & 4 & -3 & -1 & 2 \\
1 & -3 & 2 & -3 & 1 & 4 & 0 & 1 & -1 & 3
\end{array}\right]\left[\begin{array}{c}
x_{1} \\
x_{2} \\
x_{3} \\
x_{4} \\
x_{5} \\
x_{6} \\
x_{7} \\
x_{8} \\
x_{9} \\
x_{10}
\end{array}\right]=\left[\begin{array}{c}
-1 \\
11 \\
9 \\
16 \\
6 \\
9 \\
4 \\
7 \\
2 \\
6
\end{array}\right] .
$$

\section{Solution:}

Here $n=10$ and $m=5$. Using the procedure centrosymm, we get the following results:

$$
\begin{aligned}
P & =\left[\begin{array}{ccccc}
4 & -4 & 3 & -3 & 5 \\
4 & 2 & -3 & 3 & -3 \\
2 & 3 & 3 & 0 & 6 \\
4 & 2 & 0 & 2 & 3 \\
1 & 4 & 1 & 0 & 5
\end{array}\right], \quad Q=\left[\begin{array}{ccccc}
2 & 2 & -1 & 3 & 3 \\
0 & -4 & -3 & 5 & -1 \\
0 & -3 & 1 & -4 & 0 \\
2 & 4 & -6 & 6 & -5 \\
1 & -2 & 3 & -2 & 1
\end{array}\right], \\
\hat{\boldsymbol{b}} & =[5,13,16,20,15]^{\mathrm{T}} \text { and } \tilde{\boldsymbol{b}}=[-7,9,2,12,-3]^{\mathrm{T}}, \quad|R|=|P| \times|Q|=-730912 .
\end{aligned}
$$

The solutions of the systems

$$
\left[\begin{array}{ccccc}
4 & -4 & 3 & -3 & 5 \\
4 & 2 & -3 & 3 & -3 \\
2 & 3 & 3 & 0 & 6 \\
4 & 2 & 0 & 2 & 3 \\
1 & 4 & 1 & 0 & 5
\end{array}\right]\left[\begin{array}{l}
y_{1} \\
y_{2} \\
y_{3} \\
y_{4} \\
y_{5}
\end{array}\right]=\left[\begin{array}{c}
5 \\
13 \\
16 \\
20 \\
15
\end{array}\right]
$$

and

$$
\left[\begin{array}{ccccc}
2 & 2 & -1 & 3 & 3 \\
0 & -4 & -3 & 5 & -1 \\
0 & -3 & 1 & -4 & 0 \\
2 & 4 & -6 & 6 & -5 \\
1 & -2 & 3 & -2 & 1
\end{array}\right]\left[\begin{array}{c}
z_{1} \\
z_{2} \\
z_{3} \\
z_{4} \\
z_{5}
\end{array}\right]=\left[\begin{array}{c}
-7 \\
9 \\
2 \\
12 \\
-3
\end{array}\right]
$$

are $\boldsymbol{y}=[2,1,-1,2,2]^{\mathrm{T}}$ and $\boldsymbol{z}=[0,-1,-1,0,-2]^{\mathrm{T}}$.

Therefore, we have

$$
\begin{aligned}
& x_{1}=\frac{1}{2}\left(y_{1}+z_{1}\right)=1, \quad x_{6}=\frac{1}{2}\left(y_{5}-z_{5}\right)=2, \\
& x_{2}=\frac{1}{2}\left(y_{2}+z_{2}\right)=0, \quad x_{7}=\frac{1}{2}\left(y_{4}-z_{4}\right)=1, \\
& x_{3}=\frac{1}{2}\left(y_{3}+z_{3}\right)=-1, \quad x_{8}=\frac{1}{2}\left(y_{3}-z_{3}\right)=0,
\end{aligned}
$$

$$
\begin{aligned}
& x_{4}=\frac{1}{2}\left(y_{4}+z_{4}\right)=1, \quad x_{9}=\frac{1}{2}\left(y_{2}-z_{2}\right)=1, \\
& x_{5}=\frac{1}{2}\left(y_{5}+z_{5}\right)=0, \quad x_{10}=\frac{1}{2}\left(y_{1}-z_{1}\right)=1 .
\end{aligned}
$$

Hence the solution vector is

$$
\boldsymbol{x}=[1,0,-1,1,0,2,1,0,1,1]^{\mathrm{T}} .
$$

Example 6.2. Solve the centrosymmetric linear system $\left[\begin{array}{lllllllllll}2 & 1 & 0 & 0 & 0 & 0 & 0 & 0 & 0 & 0 & 0 \\ 1 & 2 & 1 & 0 & 0 & 0 & 0 & 0 & 0 & 0 & 0 \\ 0 & 1 & 2 & 1 & 0 & 0 & 0 & 0 & 0 & 0 & 0 \\ 0 & 0 & 1 & 2 & 1 & 0 & 0 & 0 & 0 & 0 & 0 \\ 0 & 0 & 0 & 1 & 2 & 1 & 0 & 0 & 0 & 0 & 0 \\ 0 & 0 & 0 & 0 & 1 & 2 & 1 & 0 & 0 & 0 & 0 \\ 0 & 0 & 0 & 0 & 0 & 1 & 2 & 1 & 0 & 0 & 0 \\ 0 & 0 & 0 & 0 & 0 & 0 & 1 & 2 & 1 & 0 & 0 \\ 0 & 0 & 0 & 0 & 0 & 0 & 0 & 1 & 2 & 1 & 0 \\ 0 & 0 & 0 & 0 & 0 & 0 & 0 & 0 & 1 & 2 & 1 \\ 0 & 0 & 0 & 0 & 0 & 0 & 0 & 0 & 0 & 1 & 2\end{array}\right]\left[\begin{array}{c}x_{1} \\ x_{2} \\ x_{3} \\ x_{4} \\ x_{5} \\ x_{6} \\ x_{7} \\ x_{8} \\ x_{9} \\ x_{10} \\ x_{11}\end{array}\right]=\left[\begin{array}{c}2 \\ 1 \\ 1 \\ 3 \\ 4 \\ 3 \\ 1 \\ 1 \\ 3 \\ 4 \\ 3\end{array}\right]$.

Solution: Here $n=11$ and $m=5$. Using the procedure centrosymm, we obtain the following results: 


$$
\begin{aligned}
& P=\left[\begin{array}{llllll}
2 & 1 & 0 & 0 & 0 & 0 \\
1 & 2 & 1 & 0 & 0 & 0 \\
0 & 1 & 2 & 1 & 0 & 0 \\
0 & 0 & 1 & 2 & 1 & 0 \\
0 & 0 & 0 & 1 & 2 & 2 \\
0 & 0 & 0 & 0 & 1 & 2
\end{array}\right], Q=\left[\begin{array}{ccccc}
2 & 1 & 0 & 0 & 0 \\
1 & 2 & 1 & 0 & 0 \\
0 & 1 & 2 & 1 & 0 \\
0 & 0 & 1 & 2 & 1 \\
0 & 0 & 0 & 1 & 2
\end{array}\right], \\
& \hat{\boldsymbol{b}}=[5,5,4,4,5,3]^{\mathrm{T}} \text { and } \tilde{\boldsymbol{b}}=[-1,-3,-2,2,3]^{\mathrm{T}}, \\
& |R|=|P| \times|Q|=12 .
\end{aligned}
$$

The solutions of the systems

$$
\left[\begin{array}{llllll}
2 & 1 & 0 & 0 & 0 & 0 \\
1 & 2 & 1 & 0 & 0 & 0 \\
0 & 1 & 2 & 1 & 0 & 0 \\
0 & 0 & 1 & 2 & 1 & 0 \\
0 & 0 & 0 & 1 & 2 & 2 \\
0 & 0 & 0 & 0 & 1 & 2
\end{array}\right]\left[\begin{array}{l}
y_{1} \\
y_{2} \\
y_{3} \\
y_{4} \\
y_{5} \\
y_{6}
\end{array}\right]=\left[\begin{array}{c}
5 \\
5 \\
4 \\
4 \\
5 \\
3
\end{array}\right]
$$

and

$$
\left[\begin{array}{lllll}
2 & 1 & 0 & 0 & 0 \\
1 & 2 & 1 & 0 & 0 \\
0 & 1 & 2 & 1 & 0 \\
0 & 0 & 1 & 2 & 1 \\
0 & 0 & 0 & 1 & 2
\end{array}\right]\left[\begin{array}{c}
z_{1} \\
z_{2} \\
z_{3} \\
z_{4} \\
z_{5}
\end{array}\right]=\left[\begin{array}{c}
-1 \\
-3 \\
-2 \\
2 \\
3
\end{array}\right]
$$

are $\boldsymbol{y}=[2,1,1,1,1,1]^{\mathrm{T}}$ and $\mathbf{z}=[0,-1,-1,1,1]^{\mathrm{T}}$.

Therefore, we get

$$
\begin{aligned}
& x_{1}=\frac{1}{2}\left(y_{1}+z_{1}\right)=1, \quad x_{7}=\frac{1}{2}\left(y_{5}-z_{5}\right)=0, \\
& x_{2}=\frac{1}{2}\left(y_{2}+z_{2}\right)=0, \quad x_{8}=\frac{1}{2}\left(y_{4}-z_{4}\right)=0 . \\
& x_{3}=\frac{1}{2}\left(y_{3}+z_{3}\right)=0, \quad x_{9}=\frac{1}{2}\left(y_{3}-z_{3}\right)=1 . \\
& x_{4}=\frac{1}{2}\left(y_{4}+z_{4}\right)=1, \quad x_{10}=\frac{1}{2}\left(y_{2}-z_{2}\right)=1 . \\
& x_{5}=\frac{1}{2}\left(y_{5}+z_{5}\right)=1, \quad x_{11}=\frac{1}{2}\left(y_{1}-z_{1}\right)=1 . \\
& x_{6}=y_{6}=1,
\end{aligned}
$$

Hence the solution vector is

$$
\boldsymbol{x}=[1,0,0,1,1,1,0,0,1,1,1]^{\mathrm{T}} .
$$

Example 6.3. Solve the centrosymmetric linear system

$$
\left[\begin{array}{cccccccccc}
1 & 0 & -1 & 2 & 3 & -2 & 1 & 0 & 4 & 1 \\
2 & 1 & 3 & -1 & 6 & 6 & -1 & 3 & 1 & 2 \\
3 & 2 & 1 & 0 & -1 & 2 & 1 & 1 & 3 & 4 \\
4 & 3 & 2 & 1 & 0 & 1 & 3 & 4 & 0 & 1 \\
5 & 4 & 3 & 0 & 1 & 2 & 4 & 5 & 1 & 3 \\
3 & 1 & 5 & 2 & 2 & 1 & 2 & 3 & 4 & 5 \\
1 & 0 & 4 & 3 & 1 & 0 & 1 & 2 & 3 & 4 \\
4 & 3 & 1 & 1 & 2 & -1 & 0 & 1 & 2 & 3 \\
2 & 1 & 3 & -1 & 6 & 6 & -1 & 3 & 1 & 2 \\
1 & 4 & 0 & 1 & -2 & 3 & 2 & -1 & 0 & 1
\end{array}\right]\left[\begin{array}{c}
x_{1} \\
x_{2} \\
x_{3} \\
x_{4} \\
x_{5} \\
x_{6} \\
x_{7} \\
x_{8} \\
x_{9} \\
x_{10}
\end{array}\right]=\left[\begin{array}{c}
-1 \\
2 \\
1 \\
3 \\
0 \\
3 \\
1 \\
1 \\
4 \\
3
\end{array}\right] .
$$

\section{Solution:}

Here $n=10$ and $m=5$. Using the procedure centrosymm, we obtain the following results:

Error Singular Matrix !!!! This means that the given system has no solutions.

\section{REFERENCES}

[1] A. L. Andrew, "Centrosymmetric Matrices," SIAM Review, Vol. 40, No. 3, 1998, pp. 697-699. http://dx.doi.org/10.1137/S0036144597328341

[2] A. Cantoni and P. Butler, "Eigenvalues and Eigenvectors of Symmetric Centrosymmetric Matrices,” Linear Algebra and Its Applications, Vol. 13, No. 3, 1976, pp. 275288.

[3] A. Cantoni and P. Butler, "Properties of the Eigenvectors of Persymmetric Matrices with Applications to Communication Theory," IEEE Transactions on Communications, Vol. 24, No. 8, 1976, pp. 804-809. http://dx.doi.org/10.1109/TCOM.1976.1093391

[4] W. Chen, Y. Yu and X. Wang, "Reducing the Computational Requirement of Differential Quadrature Method," Numerical Methods for Partial Differential Equations, Vol. 12, 1996, pp. 565-577.

[5] L. Datta and S. Morgera, "Some Results on Matrix Symmetries and a Pattern Recognition Application," IEEE Transactions on Signal Processing, Vol. 34, No. 4, 1986, pp. 992-994.

[6] L. Datta and S. Morgera, "On the Reducibility of Centrosymmetric Matrices-Applications in Engineering Problems," Circuits, Systems and Signal Processing, Vol. 8, No. 1, 1989, pp. 71-96.

[7] J. Delmas, "On Adaptive EVD Asymptotic Distribution of Centro-Symmetric Covariance Matrices,” IEEE Transactions on Signal Processing, Vol. 47, No. 5, 1999, pp. 1402-1406.

[8] I. J. Good, "The Inverse of a Centrosymmetric Matrix," Technometrics, Vol. 12, No. 4, 1970, pp. 925-928.

[9] Z.-Y. Liu, "Some Properties of Centrosymmetric Matrices," Applied Mathematics and Computation, Vol. 141, No. 2-3, 2003, pp. 297-306.

[10] R. B. Mattingly, "Even Order Regular Magic Squares Are Singular," The American Mathematical Monthly, Vol. 
107, No. 9, 2000, pp. 777-782.

[11] F. Stenger, “Matrices of Sinc Methods," Journal of Computational and Applied Mathematics, Vol. 86, No. 1, 1997, pp. 297-310.

[12] J. Weaver, "Centrosymmetric (Cross-Symmetric) Matrices, Their Basic Properties, Eigenvalues, and Eigenvectors," The American Mathematical Monthly, Vol. 92, No. 10, 1985, pp. 711-717.

[13] A. C. Aitken, "Determinants and Matrices," Oliver and Boyd, Edinburgh, 1956.

[14] H.-T. Gao, C.-H. You and Y. Yang, “An Iterative Method for Generalized Centro-Symmetric Solution of Matrix Equation $A X A^{T}+B Y B^{T}=C$, ” 2008. http://www.paper.edu.cn/en_releasepaper/content/21924.

[15] A. Graovac, O. Ori, M. Faghani and A. R. Ashrafi, "Distance Property of Fullerenes," Iranian Journal of Mathematical Chemistry, Vol. 2, No. 1, 2011, pp. 99-107.

[16] I. T. Abu-Jeib, "Algorithms for Centrosymmetric and Skew-Centrosymmetric Matrices,” Missouri Journal of Mathematical Sciences, Vol. 18, No. 1, 2006, pp. 46-53.

[17] O. Krafft and M. Schaefer, “Centrogonal Matrices,” Linear Algebra and its Applications, Vol. 306, No., 2000, pp. 145-154.

[18] Z.-Y. Liu, "Some Properties of Centrosymmetric Matrices and Its Applications," Numerical Mathematics: A Journal of Chinese Universities, Vol. 14, No. 2, 2005, pp. 136-148.

[19] Z. Tian and C. Gu, "The Iterative Methods for Centrosymmetric Matrices," Applied Mathematics and Computation, Vol. 187, No. 2, 2007, pp. 902-911.

[20] R. Vein and P. Dale, "Determinants and Their Applications in Mathematical Physics," Springer, New York, 1999.

[21] A. M. Yasuda, "Some Properties of Commuting and AntiCommuting m-Involutions,” Acta Mathematica Scientia,
Series B (English Edition), Vol. 32, No. 2, 2012, pp. 631-644.

[22] M. E. A. El-Mikkawy, “A Generalized Symbolic Thomas algoriThm,” Applied Mathematics, Vol. 3, No. 4, 2012, pp. 342-345. http://dx.doi.org/10.4236/am.2012.34052

[23] T. Sugimoto, "On an Inverse Formula of a Tridiagonal Matrix,” Operators and Matrices, Vol. 6, No. 3, 2012, pp. 465-480. http://dx.doi.org/10.7153/oam-06-30

[24] M. E. A. El-Mikkawy, "A Note on a Three-Term Recurrence for a Tridiagonal Matrix," Journal of Applied Mathematics and Computing, Vol. 139, No. 2-3, 2003, pp. 503-511.

[25] M. E. A. El-Mikkawy, "An Algorithm for Solving Tridiagonal Systems," Journal of Institute of Mathematics and Computer Science, Vol. 4, No. 2, 1991, pp. 205-210.

[26] M. E. A. El-Mikkawy, "On the Inverse of a General Tridiagonal Matrix," Applied Mathematics and Computation, Vol. 150, No. 3, 2004, pp. 669-679.

[27] M. E. A. El-Mikkawy, "A New Computational Algorithm for Solving Periodic Tri-Diagonal Linear Systems,” Applied Mathematics and Computation, Vol. 161, No. 2, 2005, pp. 691-696.

[28] M. E. A. El-Mikkawy and A. Karawia, "Inversion of General Tridiagonal Matrices,” Applied Mathematics Letters, Vol. 19, No. 8, 2006, pp. 712-720.

[29] M. E. A. El-Mikkawy and E.-D. Rahmo, “A New Recursive Algorithm for Inverting General Tridiagonal and Anti-Tridiagonal Matrices,” Applied Mathematics and Computation, Vol. 204, No. 1, 2008, pp. 368-372.

[30] R. L. Burden and J. D. Faires, "Numerical Analysis,” 7th ed., Books \& Cole Publishing, Pacific Grove, 2001.

[31] M. E. A. El-Mikkawy, “A Fast Algorithm for Evaluating nth Order Tri-Diagonal Determinants,” Journal of Computational and Applied Mathematics, Vol. 166, No. 2, 2004, pp. 581-584. 